\title{
Effects of caffeine on sleep quality and daytime functioning
}

This article was published in the following Dove Press journal: Risk Management and Healthcare Policy

\author{
Frances O'Callaghan' \\ Olav Muurlink2,3 \\ Natasha Reid ${ }^{4}$ \\ 'School of Applied Psychology, \\ Griffith Health, Griffith University, \\ Southport, QLD, Australia; ${ }^{2}$ School of \\ Business and Law, Central Queensland \\ University, Brisbane, QLD, Australia; \\ ${ }^{3} \mathrm{Griffith}$ Institute for Educational \\ Research, Griffith University, \\ Southport, QLD, Australia; ${ }^{4}$ Child \\ Health Research Centre, Faculty of \\ Medicine, University of Queensland, \\ Brisbane, QLD, Australia
}

\begin{abstract}
Caffeine (particularly in the form of coffee) is one of the most widely consumed stimulants in the world, with $90 \%$ of American adults consuming caffeine-infused beverages almost daily. While there is substantial evidence that caffeine enhances performance, caffeine withdrawal leads to deficits at both the individual (eg, cognitive, emotional, and behavioral processes) and societal (eg, increases in work accidents) level. Scholars for some time have considered that the supposed psychoactive benefits of caffeine may be the result of the mere reversal of deleterious effects of caffeine withdrawal, rather than a net benefit of caffeine ingestion. In this integrative review, we examine evidence illuminating the relationship between caffeine consumption and subsequent quality and quantity of nighttime rest. Secondly, we consider evidence as to whether performance deficits caused by sleep deprivation linked to caffeine can be reversed by caffeine consumption during the subsequent daytime period. Finally, we consider how these two stages can be reconciled in a single model that enables calculation of the net caffeine effect on daytime functioning. The literature highlights a range of positive impacts of caffeine consumption on both physical and cognitive functioning. There are also a number of factors that complicate any conclusions that can be drawn regarding the potential of caffeine to improve performance. Most critically, performance improvements the next day may simply be a result of the reversal of caffeine withdrawal. Animal studies and well-controlled human studies involving high habitual and low habitual users tend to confirm a "net benefit" for caffeine use. Further research, particularly with (necessarily rare) caffeine-naive populations, is required to elucidate the complexities of the relationship between caffeine, sleep, and daytime functioning. However, the convenience of accessing caffeine compared to ensuring adequate restorative sleep means that caffeine has applied advantages that are likely to see its use as a performance "enhancing" substance persist.
\end{abstract}

Keywords: caffeine, daytime functioning, sleep, sleep deprivation

\section{Introduction}

Caffeine (1,3,7-trimethylxanthine), particularly in the form of coffee, has become one of the most widely consumed and geographically distributed ergogenic aids, and is rated as the most widely consumed stimulant in the world. ${ }^{1}$ In the United States, for example, $90 \%$ of adults consume caffeine-infused beverages (ie, coffee, tea, energy, or other drinks) almost daily ${ }^{2}$ with consumption averaging $200 \mathrm{mg} /$ day. ${ }^{3}$ Self-report data on caffeine consumption tend to underreport actual levels due to the confounding of "coffee" and "caffeine" — with other sources, such as analgesics including cold remedies, hot chocolate, tea, and energy drinks, often overlooked. ${ }^{4}$ For example, Wendte et $a l^{5}$ painstakingly documented the habitual intake of their participants, selecting those
Correspondence: Frances O'Callagha School of Applied Psychology, Griffith University, Parklands Drive, Southport, QLD 4222, Australia

Email f.ocallaghan@griffith.edu.au 
who regularly ingested from 100 to $500 \mathrm{mg}$ of caffeine a day, finding an actual consumption range of 154-1285 mg. Such is the ubiquity of caffeine that it provides methodological challenges in terms of investigating its effects: it is difficult to provide a truly caffeine-free $\operatorname{diet}^{6}$ and given that the vast majority of people in countries such as the United States ingest caffeine of various forms almost daily, ${ }^{2}$ few research participants can present to a laboratory as truly caffeine naive.

The popularity of caffeine is driven not only by taste, given that it is a common additive in the modern diet, ${ }^{7}$ but also by its reputation as a stimulant. A study by Desbrow and Leveritt ${ }^{8}$ also highlights the power associated with this reputation as a stimulant. They report that most athletes believe that caffeine improves both endurance and concentration. Notwithstanding lay perceptions, the evidence of a relationship between caffeine and performance is, however, substantial. The ability of caffeine to enhance physical performance was formally identified early in the 20 th century ${ }^{9}$ and since then scholarly and military interest in the role of nutrition on physical performance has been intense. A comprehensive review by the Military Nutrition Division of the US Army Research Institute of Environmental Medicine found that of the nutritional components to have the clearest identifiable benefits, caffeine stands out most clearly as being a positive. ${ }^{10}$

Pharmacologically, caffeine is an adenosine-receptor antagonist. ${ }^{11}$ As such, it appears that the effects of caffeine on performance occur largely through its occupation of adenosine receptors. ${ }^{12}$ It acts primarily on $\mathrm{A} 1$ and $\mathrm{A} 2 \mathrm{~A}$ receptors, which in turn are related to functions of the brain associated with sleep, arousal, and cognition. ${ }^{13}$ Caffeine is efficiently and quickly absorbed by the stomach and small intestine, with peak plasma levels occurring in the first $30 \mathrm{~min} .{ }^{14}$ Caffeine has a highly variable half-life, ranging from 2 to $10 \mathrm{~h},{ }^{15,16}$ dependent on endogenous and exogenous factors. ${ }^{17}$ Nicotine use, for example, can increase the metabolic speed of caffeine by as much as $50 \% .{ }^{18}$ This short half-life, however, may enable caffeine to be used strategically to enhance daytime functioning with an impact on sleep quality that could be predicted and mitigated.

The residual effects, however, may be significant. Shi et $\mathrm{al}^{19}$ demonstrated that tolerance depends on the amount of caffeine consumed, and the schedule of consumption and elimination. They use a parametric pharmacokinetic-pharmacodynamic model to suggest that it can take up to $20 \mathrm{~h}$ (or the equivalent of four or five half-lives) for the effects of caffeine tolerance to wear off.

Caffeine is placed in an unusual juxtaposition in relation to human performance: it clearly has the potential to enhance performance, but amongst its known side effects are sleep deprivation, which brings with it a risk of performance deficits. In this review, we examine this balance in the context of daytime functioning. The key question that we seek to answer is: can caffeine undo the harm it potentially causes through reducing the quality of overnight sleep? That is, can performance be sustained into a second day, subsequent to a night of sleep impaired through caffeine consumption, through the administration of caffeine?

We explore this question in two distinct phases. First, we examine evidence illuminating the relationship between caffeine consumption and subsequent quality and quantity of nighttime rest. Second, we consider evidence as to whether performance deficits caused by sleep deprivation linked to caffeine can be reversed by caffeine consumption during the subsequent daytime period (see Figure 1). Finally, we consider how these two stages can be reconciled in a single model that enables the effect of caffeine on daytime to be considered.

\section{The sleep "sandwich"}

For the majority of adults in Western countries such as the United States, sandwiched between one day of caffeine consumption and the next is a period of caffeine deprivationsleep. If caffeine consumption is not wisely regulated during the first daytime, sleep deprivation will ensue, and performance deficits will be experienced during the subsequent daytime. Caffeine consumption by day causes a reduction in 6-sulfatoxymelatonin (the main metabolite of melatonin) on the ensuing night, ${ }^{20}$ which is one of the mechanisms by which sleep is interrupted. Given that the number of Americans who sleep fewer than $6 \mathrm{~h}$ per night increased from $13 \%$ to $20 \%$

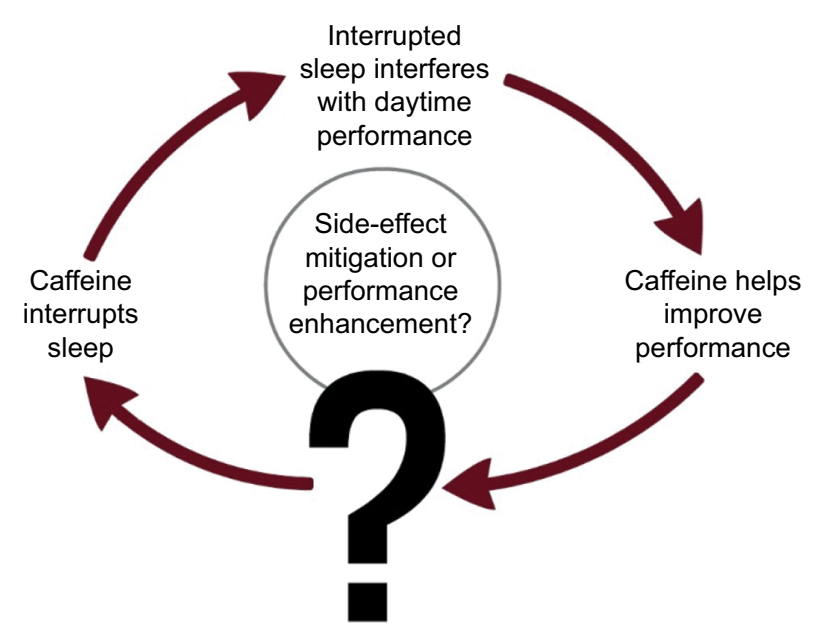

Figure I The cycle of benefits/disbenefits in caffeine consumption. 
from 1999 to 2009, widespread caffeine consumption may well have broad societal implications. ${ }^{1,21}$

Sleep deprivation is known to lead to significant decrements in cognitive function, including lapses of attention, alertness, vigilance, and the speed of cognitive and psychomotor responses..$^{22}$ Laboratory studies show that a deficit in nocturnal sleep of as little as $90 \mathrm{~min}$ for just one night can lead to a reduction of daytime objective alertness by one-third. ${ }^{23}$

Higher order processes are also implicated, including reduced cognitive control, ${ }^{24}$ disruption to planning capacity, ${ }^{25}$ ethical misbehavior, ${ }^{26}$ increased risk taking, ${ }^{27}$ altered leadership style ${ }^{28}$, and possibly even reduced creativity. Some of these higher order changes are explicable from a neuroscience perspective; sleep deprivation impairs brain functioning in the prefrontal cortex, associated with executive control over behavior. ${ }^{22}$ The relationship appears to be mediated significantly by blood glucose since sleep deprivation reduces the prefrontal cortex's rate of glucose metabolic activity. ${ }^{29}$ As such, impulse crimes and lapses of judgment are more common in the evening, and self-control is at its strongest in the morning. ${ }^{30}$ By contrast, sufficient good quality sleep appears to be conducive to both improved cognitive and physical performance that can be related to metabolic function in the brain $^{31}$ and the body itself. ${ }^{32}$

These deficits flow through to impacts measurable at a societal level, including losses in productivity, ${ }^{33}$ increases in work accidents and injuries, ${ }^{34}$ and industrial and motor accidents. ${ }^{35}$ Suboptimal sleep also can have negative impacts on social processes: it can reduce the ability of individuals to recognize emotions in facial expressions ${ }^{36}$ and increase emotional reactivity, including greater likelihood of neutral stimuli being assigned negative emotional valence. ${ }^{36}$

\section{The impact of caffeine consumption on subsequent sleep quality}

Large cross-sectional studies, such as Orbeta et al's ${ }^{37}$ examination of 15,686 American adolescents, find that after adjusting for socioeconomic factors, students reporting high caffeine intake are more likely to be tired in the morning than those reporting very low intakes. Caffeine's reputation as a performance stimulant that can reverse the deficits associated with fatigue means that it is often ingested by those who are fatigued, at the same time as being implicated in causing the fatigue in the first place.

Caffeine's reputation, of course, means that the substance carries significant expectancy effects, with Sun et a $\mathrm{l}^{38}$ finding that an initial dose of caffeine followed by a placebo $4 \mathrm{~h}$ later helped extend the cognitive performance of the initial caffeine dose beyond the period when elevated blood pressure and heart rate subsided. However, the Atahualpa Project, using data collected in an Ecuadoran village with no fast food outlets, almost no shift work, and little light or noise pollution during the evening and night hours - and almost certainly a very different cultural orientation toward caffeine - found no impact of caffeine consumption on sleep patterns when controlling for other physiological variables. ${ }^{39}$ The researchers note that the absence of caffeine impact on night sleep quality may be associated with the absence of other secondary triggers to wakefulness, such as noise or artificial light.

This complicates an analysis of causation. Researchers have commonly found that feeling tired in the morning leads to high caffeine use, which in turn is associated with impaired subsequent sleep patterns; ${ }^{17,40}$ what might be called a "coffee cycle". Drake et $\mathrm{al}^{41}$ examined the effect of $400 \mathrm{mg}$ of caffeine administered at three points prior to their participants' usual bedtime, and found that doses even $6 \mathrm{~h}$ prior to bedtime significantly disturbed sleep compared to placebos. The study also found no difference in total sleep time (TST) between the conditions where caffeine was taken immediately before bed or $3 \mathrm{~h}$ prior to bedtime. With $400 \mathrm{mg}$ being equivalent to four cups of brewed coffee, the study used a relatively high dose, with the impact most felt in TST. Karacan et al's classic study ${ }^{42}$ took a different approach, using a single population and varying doses of caffeine administered 30 min prior to bedtime in a balanced Latin-square design, finding the impact on TST was dose related. Caffeine shifted rapid eye movement to the early part of the night and stages 3 and 4 to the latter end of the (shortened) sleep period. Most studies have examined caffeine administration during the daytime with large latencies between ingestion and sleep onset, which of course allows interactions with other potential sleep interference vectors such as dietary intake.

Landolt et $\mathrm{a}^{43}$ investigated the role of caffeine in sleep regulation, with participants administered $200 \mathrm{mg}$ of caffeine in the early morning ( $7 \mathrm{am}$ ) and then monitored using electroencephalography. Caffeine levels in the saliva were observed to spike $1 \mathrm{~h}$ after intake, falling to less than a fifth of that level $(3 \mu \mathrm{mol} / \mathrm{L}) 16 \mathrm{~h}$ later. Despite this reduction at sleep onset, both sleep efficiency and TST were significantly reduced in experimental conditions, relative to placebo. The presence of caffeine in the central nervous system, the authors concluded, reduces the gradual onset of drowsiness associated with extended periods of wakefulness.

It is possible that people learn to regulate consumption to fit their individual patterns of response. A study of 2,202 
randomly selected residents of three European nations (Iceland, Sweden, and Belgium) gathered data on sleep characteristics and the use of psychoactive substances including coffee. ${ }^{44}$ They found that caffeine consumption did not predict difficulties inducing sleep or other sleep disturbances when age, gender, smoking, and seasonal variations were controlled for.

So, the impact of caffeine consumption on subsequent sleep quality is complicated by a number of factors. For example, it is often ingested by those who are already fatigued, as well as being implicated in causing the fatigue in the first place. Research by del Brutto et $\mathrm{al}^{39}$ noted secondary triggers to wakefulness, such as noise and artificial light, as well as interactions with other factors such as diet which may interfere with sleep.

\section{The impact of caffeine deprivation and withdrawal}

Due to the prevalence of caffeine in the "normal" diet of most adults presenting for studies, it is useful to examine the findings in studies where caffeine is eliminated from the diet, and absence of caffeine is verified with blood plasmaconsidered a good indicator of caffeine concentrations in the brain. ${ }^{45}$ Relatively few sleep (let alone sleep and performance) studies examine this outcome. Thus, it is difficult to map the net effects of caffeine from the current literature, because almost all studies do not look just at an absence of caffeine, but inevitably incorporate caffeine withdrawal. Caffeine withdrawal has been shown to have its own correlates in terms of decreased performance in both humans ${ }^{46}$ and animals. ${ }^{47}$ One of the landmark studies in the field, a double-blind study involving the sudden cessation of caffeine intake from a mean daily dose of $235 \mathrm{mg}$, resulted in moderate to severe headaches, increases in anxiety and depression, and reduced speed of simple motor tasks (finger tapping). ${ }^{46}$

The literature generally suggests that tolerance to caffeine develops with sustained use. ${ }^{48}$ However, Debrah et $\mathrm{a}^{49}$ have demonstrated that tolerance develops in peripheral blood pressure but not in cerebral blood flow-from which the performance benefits are likely to issue. This suggests that some of the performance benefits of caffeine might be sustainable. What complicates the issue further, however, is that with such a short half-life, caffeine deprivation occurs each night for regular coffee users. The work of Richardson's team in the Institute of Food Research in Reading ${ }^{50}$ suggests that acute intake of caffeine impacted similarly on experimental participants who were relatively heavy users, and those who consumed little or no caffeine. Richardson et $\mathrm{al}^{50}$ examined deprivation periods ranging from $90 \mathrm{~min}$, overnight, to a week on young adults who were either regular moderate or nonconsumers of caffeine products. Even overnight caffeine deprivation produced negative impacts on mood that could be reversed through caffeine consumption - and mood regulation may influence coffee consumption. Acute caffeine intake affected participants in different categories equally - those caffeine-deprived for a week responded similarly to those deprived for shorter periods. Caffeine consumption increased jitteriness, but decreased headaches and fatigue, with hand steadiness increasing on a dose-dependent basis. Reaction time increased, but only at lower doses.

By contrast, a more recent study made the same comparison between relatively high users and those who were close to caffeine naive but focused on performance within a single day after a night of withdrawal. Rogers et $\mathrm{al}^{51}$ used saliva tests to confirm consumption status and looked at performance improvements following caffeine ingestion after a night of abstinence in both groups. They found that in the morning, those who had experienced a withdrawal overnight did perform worse on reaction time tasks and were marginally less alert, suggesting withdrawal had made them worse off than they would otherwise have been. The anxiety/jitteriness impacts of ingesting caffeine were more pronounced, however, for those who were noncaffeine or low caffeine consumers than on those accustomed to caffeine, suggesting regular users had become accustomed to the nightly withdrawal pattern.

Rogers and his team recognize that differences between the groups may reflect nonrandom effects of self-selection into caffeine or noncaffeine conditions. Those prone to morning sleepiness, for example, may be more likely to choose to ingest caffeine. While studies of caffeine consumption and deprivation in humans are hampered by these constraints, animal studies do not suffer this limitation. The finding there, while tending to support the notion of a net positive despite withdrawal decrements, is that the relationships between caffeine and performance are complex and task specific. ${ }^{52}$

\section{Reversal of sleep-deprivation performance deficits through the use of caffeine}

Most studies looking at improving performance using caffeine ironically make use of sleep disruption at least partially induced by caffeine (even introducing caffeine intravenously during sleep $)^{53}$ to show their effects. Studies rarely properly control for caffeine presence, particularly objectively measured through blood plasma levels. 
Having consumed caffeine to stay awake and perform optimally, in subsequent days the consumer may pay the penalty. This assumption can easily be "supported" through cross-sectional research such as that conducted by Pecotic et al ${ }^{54}$ who asked 323 medical professionals about their sleep habits and caffeine use, finding that those who consumed more caffeine reported having greater trouble staying awake. Feeling tired in the morning induces higher caffeine ingestion. ${ }^{40}$ Caffeine consumption may equally be a response to fatigue as a cause of it. ${ }^{37}$

Tieges et a $1^{55}$ examined accuracy of action monitoring of a relatively simple task, finding that caffeine doses of 3 and $5 \mathrm{mg} / \mathrm{kg}$ of body weight (considered moderate) lead to a reduction in errors relative to a placebo condition. In a recent study, Faber et al ${ }^{56}$ plausibly suggest that improvements in caffeine-related performance might be moderated by group effects, with elevated arousal associated with higher doses leading to reduced group performance, but present no primary evidence to test their complex hypotheses.

It is possible that after a period of overnight abstinence the improvements associated with caffeine ingestion observed the next day are simply the reversal of caffeine withdrawal. ${ }^{57-59}$ Rogers and Smith ${ }^{60,61}$ have been prominent in proposing the withdrawal model. The issue, then, of caffeine habituation needs to be considered. Regular intake of caffeine increases the number of adenosine receptors in the central nervous system ${ }^{17}$ with the body responding to a reduction in caffeine intake by reducing the number of adenosine receptors, a process that can take days. ${ }^{62}$ For example, in one commonly cited study, ${ }^{63}$ heavy caffeine users and "non" users were tested at a 7-day interval, with the user group maintaining consumption for the first 5 days of the testing period and then abstaining for the ensuing 2 days. Users showed a significant drop in performance at the second testing, whereas there was no significant difference at Time 1. However, the study did not (and could not) randomly assign people to the "user" and "non" user group, and this is a problem. It is possible, for example, that those experiencing sleep-related deficits in performance commonly "use" caffeine to overcome those deficits. By contrast, there are a number of studies that show that after only a few hours of "deprivation" (ie, when no significant deprivation has occurred) caffeine users will still show significant performance enhancement on ingesting caffeine, relative to placebo. ${ }^{64,65}$ Some have argued, however, that even these studies do not persuasively overcome the withdrawal case. Lara ${ }^{66}$ for example, notes that even brief abstinence is accompanied by withdrawal symptoms. Attempts to examine the interactive effect of caffeine and sleep deprivation, including many of the studies cited in this paper, by their very nature, explore that interaction.

While studies do appear to indicate a deterioration of mood, even after the relatively minor withdrawal associated with overnight caffeine deprivation, they do not tend to indicate a deterioration in other effects, such as psychomotor performance. ${ }^{51}$ Comparing high and low habitual consumers should be able to distinguish these effects. Mitchell and Redman ${ }^{67}$ hypothesized that the cancellation of withdrawal effects should be higher for habitual consumers than caffeine-naive subjects. Over a variety of different psychomotor and cognitive tasks, they failed to show this pattern. In a particularly persuasive study, Kenemans and Lorist ${ }^{68}$ examined differences in performance between 12-h caffeine-deprived subjects receiving caffeine, similarly deprived subjects administered a placebo, and nondeprived subjects who were given neither stimulant nor placebo. The task was sensory discrimination; the outcomes were both hit rate and reaction time. They argue that the difference between the deprivation/placebo and the nondeprivation conditions should indicate the extent of withdrawal effects, and differences between deprivation/ caffeine and nondeprivation conditions should give an indication of the net benefit of caffeine. This study concluded that withdrawal and net effects are both measurable, and are of comparable size for hit rates, but on reaction time the "true" caffeine effect was considerably larger. Finding true "caffeinenaive" subjects for studies in developed-world laboratories is a challenge; however, animal studies offer truly caffeine-naive analogs, and these studies also tend to confirm net benefits. ${ }^{52}$

Further, caffeine users may see elevated levels of selfdeception about their own performance skill. ${ }^{69}$ That is, they may overestimate the impact. However, interestingly, the impact of caffeine on performance appears to be independent of the perception of drowsiness. Ataka et al, ${ }^{70}$ for example, gave healthy participants either a placebo or $100 \mathrm{mg}$ /day of caffeine, then induced fatigue through a strenuous task performance. They found, like Kilpeläinen et al, ${ }^{71}$ that task performance improved without subjective perception of fatigue, motivation, or sleepiness altering.

As Snel and Lorist ${ }^{17}$ point out, it is the interaction between "task, participant, and environment that actually determines the quality of performance, especially during suboptimal situations" (p. 108), and tasks that are more engaging or stimulating may "mask" fatigue effects. Kilpeläinen et al, ${ }^{71}$ for example, argued that the absence of performance decline in their study of military pilot students, during working memory tasks, contrasted with significant impediments on less engaging requirements such as reaction time tasks. 
For those using caffeine to "push on" during night shifts, and relying on sleep the following day for recovery, a study by Carrier et $\mathrm{al}^{72}$ suggests that sleep efficiency is reduced to a greater extent than for those who take caffeine at the same hour in the evening but attempt a nighttime sleep. For both daytime recovery sleepers and those attempting to sleep during "normal" circadian hours, caffeine at night represents a risk, this study suggests; however, the risk is greater for those attempting to use caffeine to shift sleep to the following day. The circadian rhythm not only significantly "overrides" the impact of caffeine for the night sleepers, but for the night workers attempting a daytime recovery sleep, the circadian wakefulness propensity drive appears to combine (rather than overlap) with the dissipation of homeostatic sleep pressure.

Lajambe et $\mathrm{al}^{73}$ examined the question of habituation in a design that compared habitually low caffeine users against high (400 mg per day or above) users in a randomized crossover design. The participants were kept awake for $27 \mathrm{~h}$, and at 3,5 , and $7 \mathrm{~h}$ were given either repeated doses of caffeine gum at low or high doses, vs a placebo. They were woken after 8 $\mathrm{h}$ and administered the psychomotor vigilance task (PVT). In the high dose caffeine condition, there was impaired sleep maintenance and reduced sleep depth, with the lower habitual users worse affected, although postrecovery PVT performance did not differ by dose or habitual caffeine use.

Studies looking at caffeine-naive subjects are relatively rare in the literature, with even recent studies claiming caffeine-naive participants failing to adequately verify that status. ${ }^{74,75}$ This gap in the literature is partly due to the difficulty of recruiting subjects who are truly caffeine naive (and these subjects may be nonrepresentative of the population) due to the ubiquity of caffeine in the modern diet. Studies that attempt to work with participants exposed to very low levels of caffeine at baseline tend to confirm the positive impacts on physical ${ }^{76}$ and cognitive ${ }^{77}$ functioning.

Lieberman ${ }^{10}$ examined Navy Seals working with a more complete array of cognitive measures to see if caffeine could improve cognitive performance in those exposed to sustained multiple stressors, including sleep deprivation. The participants were deprived of sleep for $72 \mathrm{~h}$, and exposed continuously to other stressors. A bank of cognitive tests including visual vigilance, reaction time, working memory, and learning tasks, as well as practical tests on marksmanship, were administered 1 and $8 \mathrm{~h}$ after being dispensed either a 100 , 220 , or $300 \mathrm{mg}$ caffeine (or placebo) capsule. As anticipated, sleep deprivation severely affected performance and mood, and caffeine improvement on performance was particularly pronounced on vigilance, reaction time, and alertness, with lesser effects on acquisition, fatigue, and sleepiness. Marksmanship, which requires fine motor coordination, was not affected by caffeine. The results persisted (although were muted) at $8 \mathrm{~h}$ post administration.

Impact on marksmanship (an authentic test of performance in this case) was also investigated by Tharion et al, ${ }^{78}$ with this study showing reaction time improvements (in this case measured through sighting time with the rifle) and caffeine dose-dependent improvements. Additionally, McLellan et $\mathrm{al}^{79}$ found a caffeine advantage in marksmanship when compared with placebo. A third study in this sequence ${ }^{80}$ confirmed this pattern in a design involving similar tests (with the addition of logical reasoning tests) and personnel, but allowing two periods of 4-h afternoon sleeps during a 72-h testing period. The study showed the same pattern of response, and in addition showed a highly significant increase in response speed during logical reasoning tests throughout three overnight testing periods. The authors concluded that $800 \mathrm{mg}$ doses of caffeine each 24-h period during the highly stressful and sleep-deprived period allowed participants to maintain cognitive function.

In one placebo-controlled study, ${ }^{81}$ participants completed a bank of simulated driving tasks. There were highly significant effects of caffeine on alertness and a somewhat less striking impact on reduction in steering variability. Welsh et $\mathrm{a}^{82}$ found that caffeine moderated the relationship between sleep deprivation and deficits in certain higher order behaviors (they examined ethical behavior) by "replenishing self-regulator resources" (p. 1268)

Overall, the evidence suggests that caffeine ingestion has positive impacts on physical and cognitive functioning, although a number of issues complicate conclusions regarding the reversal of sleep-deprivation performance deficits through caffeine use. These issues include the lack of studies that objectively control for caffeine presence through blood plasma levels; the fact that caffeine consumption may equally be a response to fatigue as a cause of it; the possibility that following overnight abstinence, improvements in performance the next day may simply be due to the reversal of the impact of caffeine withdrawal; caffeine habituation; and complexities created by the interaction between the task, participant, and environment.

\section{Conclusion: integrating the findings}

Attempts to design integrated mathematical models that can predict the effects of sleep/wake schedules while incorporating adenosine-receptor antagonists like caffeine are rare. The two best validated predictive models of performance do not 
account for the interference by caffeine, ${ }^{1,83}$ while Benitez et $\mathrm{al}^{84}$ and Ramakrishnan et $\mathrm{l}^{85,86}$ attempt to incorporate caffeine, but do so by "cleaning up" the scenario by only looking at effects under conditions of total sleep deprivation. Two models have broken this trend: Puckeridge et $\mathrm{al}^{87}$ and Ramakrishnan et al. ${ }^{1}$ The more recent "unified" model ${ }^{1}$ is based on data drawn from two studies and then validated against a further five studies using a variety of populations and scenarios, but only used PVT data as a performance measure. None of the datasets used to validate the model followed normal day-night rhythms, with the focus of the model validation being on sleep rather than caffeine.

In all but one of the studies included in the creation and validation of the model, caffeine was consumed at least $14 \mathrm{~h}$ prior to sleep, meaning the accuracy in modeling the response when caffeine is taken immediately prior to bedtime is still unknown. However, the study did show that accounting for the caffeine resulted in improved predictions (by up to 70\%) suggesting that caffeine has an "override" role to play that supports the "net benefit" assumption. ${ }^{1}$ Because the studies upon which the model was based involved conditions of severe sleep deprivation, there is a limit to the value of the model in the current case, but the authors observe "these simulations ... suggest that $200 \mathrm{mg}$ of caffeine ... can elicit equivalent performance benefits [to] ... three hours of additional sleep" adding that the finding deserves additional research as "sleep extension is more logistically challenging than caffeine administration in most operational environments". ${ }^{1}$ It is this convenience factor, the "magic pill" effect, that can, in pragmatic terms, override the empirical question about net benefit. That is, caffeine offers a convenient way to overcome some of the deficits associated with sleep deprivation. The performance benefits in highly applied settings such as sport and the military appear to be widely accepted, but its ubiquity is partly related to the convenience factor.

The literature highlights a range of positive impacts of caffeine consumption on both physical and cognitive functioning. These benefits are measurable and numerous; the negative impacts, equally, are statistically and clinically significant. Studies are limited by the sheer pervasiveness of caffeine in the modern diet, and the popular consensus about the "benefits" of caffeine clearly drives some of these "benefits". A further complication is what appears to be high variability in individual response to caffeine, including the suppression of sleep. Equally, some of the "true" benefits seen in animal studies may not appear in human studies where motivation and compensation may mask the deterioration that occurs during fatigue-induced task difficulty. Evidence of this compensatory effect includes Kilpeläinen et al's ${ }^{71}$ study of military pilots where they noted relatively little performance decline in engaging tasks, relative to simpler less engaging ones.

While studies do appear to indicate a deterioration of mood, even after the relatively minor withdrawal associated with overnight caffeine deprivation, animal studies and well-controlled human studies involving high habitual and low habitual users tend to confirm a "net benefit" for caffeine use, particularly in relation to psychomotor performance. Further research, particularly with (necessarily rare) caffeine-naive populations, is required to elucidate the complexities of the relationship between caffeine, sleep, and daytime functioning. This would ideally involve an integrated double blind study, whereby a larger cohort with caffeine levels controlled and monitored through blood plasma samples were exposed to daytime and evening caffeine at various dosages to monitor impact on sleep and next-day performance. However, the convenience of accessing caffeine compared to ensuring adequate restorative sleep means that caffeine has applied advantages that are likely to see its use as a performance "enhancing" substance persist.

\section{Disclosure}

The authors report no conflicts of interest in this work.

\section{References}

1. Ramakrishnan S, Wesensten NJ, Kamimori GH, Moon JE, Balkin TJ, Reifman J. A unified model of performance for predicting the effects of sleep and caffeine. Sleep. 2016;39(10):1827-1841.

2. Mccusker RR, Goldberger BA, Cone EJ. Caffeine content of energy drinks, carbonated sodas, and other beverages. J Anal Toxicol. 2006;30(2):112-114.

3. FDA. Medicines in My Home: Caffeine and Your Body. Silver Spring: US Food and Drug Administration. 2007.

4. Brice CF, Smith AP. Effects of caffeine on mood and performance: a study of realistic consumption. Psychopharmacology. 2002;164(2):188-192.

5. Wendte R, Snel J, Tieges Z. Misclassification of caffeine use: causes and examples. In: Fac. Psychologie, Programmagroep Psychonomie. Amsterdam: Universiteit van Amsterdam; 2013:1-20.

6. Meiselman HL, Lieberman HR. Mood and performance foods. In: Functional Foods. Berlin: Springer;1994:126-150.

7. Varma SD, Kovtun S, Hegde K. Effectiveness of topical caffeine in cataract prevention: studies with galactose cataract. Mol Vis. 2010;16:2626.

8. Desbrow B, Leveritt M. Well-trained endurance athletes' knowledge, insight, and experience of caffeine use. Int J Sport Nutr Exerc Metab. 2007;17(4):328-339.

9. Rivers WH, Webber HN. The action of caffeine on the capacity for muscular work. J Physiol. 1907;36(1):33-47.

10. Lieberman HR. Nutrition, brain function and cognitive performance. Appetite. 2003;40(3):245-254.

11. Nehlig A. Coffee, Tea, Chocolate, and the Brain. Boca Raton: CRC Press; 2004.

12. van Dongen HP, Price NJ, Mullington JM, Szuba MP, Kapoor SC, Dinges DF. Caffeine eliminates psychomotor vigilance deficits from sleep inertia. Sleep. 2001;24(7):813-819. 
13. Ribeiro JA, Sebastião AM. Caffeine and adenosine. J Alzheimers Dis. 2010;20(s1):S3-S15.

14. Einöther SJ, Giesbrecht T. Caffeine as an attention enhancer: reviewing existing assumptions. Psychopharmacology. 2013;225(2):251-274.

15. Blanchard J, Sawers SJ. Comparative pharmacokinetics of caffeine in young and elderly men. J Pharmacokinet Biopharm. 1983;11(2):109-126.

16. Blanchard J, Sawers SJ. The absolute bioavailability of caffeine in man. Eur J Clin Pharmacol. 1983;24(1):93-98.

17. Snel J, Lorist MM. Effects of caffeine on sleep and cognition. In: Progress in Brain Research. Vol 190. Elsevier; 2011:105-117.

18. Wolk BJ, Ganetsky M, Babu KM. Toxicity of energy drinks. Curr Opin Pediatr. 2012;24(2):243-251.

19. Shi J, Benowitz N, Denaro C, Sheiner L. Population pharmacokinetic modeling pharmacodynamic modeling of caffeine-tolerance to pressor effects. Paper presented at: Clinical Pharmacology \& Therapeutics Ninety-third Annual Meeting; March 18-20; 1992; Orlando, FL, USA.

20. Shilo L, Sabbah H, Hadari R, et al. The effects of coffee consumption on sleep and melatonin secretion. Sleep Med. 2002;3(3):271-273.

21. Balkin T, Belenky G, Drake C, Rosa R, Rosekind M. Sleep in America poll: national sleep foundation. Washington DC: National Sleep Foundation: 2008.

22. Goel N, Rao H, Durmer JS, Dinges DF. Neurocognitive consequences of sleep deprivation. Semin Neurol. 2009;29(4):320-339.

23. Bonnet MH, Arand DL. Hyperarousal and insomnia: state of the science. Sleep Med Rev. 2010;14(1):9-15.

24. Slama H, Chylinski DO, Deliens G, Leproult R, Schmitz R, Peigneux P. Sleep deprivation triggers cognitive control impairments in task-goal switching. Sleep. Epub 2017 Dec 13.

25. Barner C, Altgassen M, Born J, Diekelmann S. Effects of sleep on the realization of complex plans. J Sleep Res. Epub 2018 Jan 10.

26. Welsh DT, Mai KM, Ellis APJ, Christian MS. Overcoming the effects of sleep deprivation on unethical behavior: an extension of integrated self-control theory. Journal of Experimental Social Psychology. 2018;76:142-154.

27. Killgore WD, Kamimori GH, Balkin TJ. Caffeine protects against increased risk-taking propensity during severe sleep deprivation. JSleep Res. 2011;20(3):395-403.

28. Carleton E. Sleep Well, Work Well: Three Studies [doctoral dissertation]. Kingston: Queen's University; 2015.

29. Gailliot MT, Baumeister RF. The physiology of willpower: linking blood glucose to self-control. In: Self-Regulation and Self-Control. Abingdon: Routledge;2018:137-180.

30. Baumeister RF, Heatherton TF, Tice DM. Losing Control: How and Why People Fail at Self-Regulation. San Diego, CA: Academic Press; 1994.

31. Thomas ML, Sing HC, Belenky G. Neural basis of alertness and cognitive performance impairments during sleepiness: II. Effects of 48 and $72 \mathrm{~h}$ of sleep deprivation on waking human regional brain activity. Thalamus \& Related Systems. 2003;2(3):199-229.

32. Schmid SM, Hallschmid M, Schultes B. The metabolic burden of sleep loss. Lancet Diabetes Endocrinol. 2015;3(1):52-62.

33. Rosekind MR, Gregory KB, Mallis MM, Brandt SL, Seal B, Lerner D. The cost of poor sleep: workplace productivity loss and associated costs. J Occup Environ Med. 2010;52(1):91-98.

34. Uehli K, Mehta AJ, Miedinger D, et al. Sleep problems and work injuries: a systematic review and meta-analysis. Sleep Med Rev. 2014;18(1): 61-73.

35. Itani O, Kaneita Y, Jike M, et al. Sleep-related factors associated with industrial accidents among factory workers and sleep hygiene education intervention. Sleep and Biological Rhythms. 2018;35:1-13.

36. Tempesta D, Couyoumdjian A, Curcio G, et al. Lack of sleep affects the evaluation of emotional stimuli. Brain Res Bull. 2010;82(1-2): 104-108.

37. Orbeta RL, Overpeck MD, Ramcharran D, Kogan MD, Ledsky R. High caffeine intake in adolescents: associations with difficulty sleeping and feeling tired in the morning. J Adolesc Health. 2006;38(4):451-453.
38. Sun Y, Zhang Y, He N, Liu X, Miao D. Caffeine and placebo expectation: effects on vigilance, cognitive performance, heart rate, and blood pressure during 28 hours of sleep deprivation. Journal of Psychophysiology. 2007;21(2):91.

39. del Brutto OH, Mera RM, Zambrano M, Castillo PR. Caffeine intake has no effect on sleep quality in community dwellers living in a rural Ecuadorian village (The Atahualpa Project). Sleep Sci. 2016;9(1):35-39.

40. Roehrs T, Roth T. Caffeine: sleep and daytime sleepiness. Sleep Med Rev. 2008;12(2):153-162.

41. Drake C, Roehrs T, Shambroom J, Roth T. Caffeine effects on sleep taken 0,3, or 6 hours before going to bed. J Clin Sleep Med. 2013;9(11):1195.

42. Karacan I, Thornby JI, Anch M, Booth GH, Williams RL, Salis PJ. Dose-related sleep disturbances induced by coffee and caffeine. Clin Pharmacol Ther. 1976;20(6):682-689.

43. Landolt HP, Werth E, Borbély AA, Dijk DJ. Caffeine intake (200 mg) in the morning affects human sleep and EEG power spectra at night. Brain Res. 1995;675(1-2):67-74.

44. Janson C, Gislason T, de Backer W, et al. Prevalence of sleep disturbances among young adults in three European countries. Sleep. 1995;18(7):589-597.

45. Kaplan GB, Greenblatt DJ, Leduc BW, Thompson ML, Shader RI. Relationship of plasma and brain concentrations of caffeine and metabolites to benzodiazepine receptor binding and locomotor activity. J Pharmacol Exp Ther. 1989;248(3):1078-1083.

46. Silverman K, Evans SM, Strain EC, Griffiths RR. Withdrawal syndrome after the double-blind cessation of caffeine consumption. N Engl J Med. 1992;327(16):1109-1114.

47. Griffiths RR, Woodson PP. Caffeine physical dependence: a review of human and laboratory animal studies. Psychopharmacology. 1988;94(4):437-451.

48. Robertson D, Wade D, Workman R, Woosley RL, Oates JA. Tolerance to the humoral and hemodynamic effects of caffeine in man. $J$ Clin Invest. 1981;67(4):1111-1117.

49. Debrah K, Haigh R, Sherwin R, Murphy J, Kerr D. Effect of acute and chronic caffeine use on the cerebrovascular, cardiovascular and hormonal responses to orthostasis in healthy volunteers. Clin Sci. 1995;89(5):475-480.

50. Richardson NJ, Rogers PJ, Elliman NA, O’Dell RJ. Mood and performance effects of caffeine in relation to acute and chronic caffeine deprivation. Pharmacol Biochem Behav. 1995;52(2):313-320.

51. Rogers P, Richardson NJ, Dernoncourt C. Caffeine use: is there a net benefit for mood and psychomotor performance? Neuropsychobiology. 1995;31(4):195-199.

52. Takahashi RN, Pamplona FA, Prediger RD. Adenosine receptor antagonists for cognitive dysfunction: a review of animal studies. Front Biosci. 2008;13(26):2614-2632.

53. Lin AS, Uhde TW, Slate SO, Mccann UD. Effects of intravenous caffeine administered to healthy males during sleep. Depress Anxiety. 1997;5(1):21-28

54. Pecotić R, Valić M, Kardum G, Sevo V, Dogas Z. Sleep habits of medical students, physicians and nurses regarding age, sex, shift work and caffein consumption. Lijec Vjesn. 2008;130(3-4):87-91.

55. Tieges Z, Richard Ridderinkhof K, Snel J, Kok A. Caffeine strengthens action monitoring: evidence from the error-related negativity. Brain Res Cogn Brain Res. 2004;21(1):87-93.

56. Faber NS, Häusser JA, Kerr NL. Sleep deprivation impairs and caffeine enhances my performance, but not always our performance: How acting in a group can change the effects of impairments and enhancements. Personality and Social Psychology Review. 2017;21(1):3-28.

57. James JE. Does caffeine enhance or merely restore degraded psychomotor performance? Neuropsychobiology. 1994;30(2-3):124-125.

58. Keenan EK, Tiplady B, Priestley CM, Rogers PJ. Naturalistic effects of five days of bedtime caffeine use on sleep, next-day cognitive performance, and mood. J Caffeine Res. 2014;4(1):13-20.

59. Heatherley SV. Caffeine withdrawal, sleepiness, and driving performance: what does the research really tell us? Nutr Neurosci. 2011;14(3):89-95. 
60. Rogers PJ. Caffeine and alertness: in defense of withdrawal reversal. Journal of Caffeine Research. 2014;4(1):3-8.

61. Rogers PJ, Smith JE. Chapter 10: Caffeine, mood and cognition. In: Benton D, editor. Lifetime Nutritional Influences on Cognition, Behaviour and Psychiatric Illness. Cambridge: Woodhead Publishing; 2011:251-271.

62. Juliano LM, Griffiths RR. A critical review of caffeine withdrawal: empirical validation of symptoms and signs, incidence, severity, and associated features. Psychopharmacology. 2004;176(1):1-29.

63. Rizzo AA, Stamps LE, Fehr LA. Effects of caffeine withdrawal on motor performance and heart rate changes. Int J Psychophysiol. 1988;6(1):9-14.

64. Frewer LJ, Lader M. The effects of caffeine on two computerized tests of attention and vigilance. Human Psychopharmacology: Clinical and Experimental. 1991;6(2):119-128.

65. Warburton DM. Effects of caffeine on cognition and mood without caffeine abstinence. Psychopharmacology. 1995;119(1):66-70.

66. Lara DR. Personality Traits and Coffee Intake. In: Preedy VR, editor. Coffee in Health and Disease Prevention. London: Academic Press; 2015. 2015:275-279.

67. Mitchell PJ, Redman JR. Effects of caffeine, time of day and user history on study-related performance. Psychopharmacology. 1992;109(1-2):121-126.

68. Kenemans JL, Lorist MM. Caffeine and selective visual processing Pharmacol Biochem Behav. 1995;52(3):461-471.

69. Baranski JV, Fatigue BJV. Fatigue, sleep loss, and confidence in judgment. J Exp Psychol Appl. 2007;13(4):182-196.

70. Ataka S, Tanaka M, Nozaki S, et al. Effects of oral administration of caffeine and D-ribose on mental fatigue. Nutrition. 2008;24(3):233-238.

71. Kilpeläinen AA, Huttunen KH, Lohi JJ, Lyytinen H. Effect of caffeine on vigilance and cognitive performance during extended wakefulness. The International Journal of Aviation Psychology. 2010;20(2):144-159.

72. Carrier J, Fernandez-Bolanos M, Robillard R, et al. Effects of caffeine are more marked on daytime recovery sleep than on nocturnal sleep. Neuropsychopharmacology. 2007;32(4):964-972.

73. Lajambe CM, Kamimori GH, Belenky G, Balkin TJ. Caffeine effects on recovery sleep following $27 \mathrm{~h}$ total sleep deprivation. Aviat Space Environ Med. 2005;76(2):108-113.

74. Cheung M, Quach J, Chan A, Nguyen NN, Shah SA. Effects of energy shots on blood pressure in caffeine-naive versus caffeine-consuming healthy volunteers. Journal of Caffeine Research. 2016;6(4):148-153.

75. Greulich S, Kaesemann P, Seitz A, et al. Effects of caffeine on the detection of ischemia in patients undergoing adenosine stress cardiovascular magnetic resonance imaging. J Cardiovasc Magn Reson. 2017;19(1):103.
76. Woolf K, Bidwell WK, Carlson AG. Effect of caffeine as an ergogenic aid during anaerobic exercise performance in caffeine naïve collegiate football players. J Strength Cond Res. 2009;23(5):1363-1369.

77. Kennedy MD, Galloway AV, Dickau LJ, Hudson MK. The cumulative effect of coffee and a mental stress task on heart rate, blood pressure, and mental alertness is similar in caffeine-naïve and caffeine-habituated females. Nutr Res. 2008;28(9):609-614.

78. Tharion WJ, Shukitt-Hale B, Lieberman HR. Caffeine effects on marksmanship during high-stress military training with 72 hour sleep deprivation. Aviat Space Environ Med. 2003;74(4):309-314.

79. Mclellan TM, Kamimori GH, Bell DG, Smith IF, Johnson D, Belenky G. Caffeine maintains vigilance and marksmanship in simulated urban operations with sleep deprivation. Aviat Space Environ Med. 2005;76(1):39-45

80. Kamimori GH, Mclellan TM, Tate CM, Voss DM, Niro P, Lieberman HR. Caffeine improves reaction time, vigilance and logical reasoning during extended periods with restricted opportunities for sleep. Psychopharmacology. 2015;232(12):2031-2042.

81. Brice C, Smith A. The effects of caffeine on simulated driving, subjective alertness and sustained attention. Hum Psychopharmacol. 2001;16(7):523-531.

82. Welsh DT, Ellis AP, Christian MS, Mai KM. Building a self-regulatory model of sleep deprivation and deception: the role of caffeine and social influence. J Appl Psychol. 2014;99(6):1268-1277.

83. Mccauley P, Kalachev LV, Mollicone DJ, Banks S, Dinges DF, van Dongen HP. Dynamic circadian modulation in a biomathematical model for the effects of sleep and sleep loss on waking neurobehavioral performance. Sleep. 2013;36(12):1987-1997.

84. Benitez PL, Kamimori GH, Balkin TJ, Greene A, Johnson ML. Modeling fatigue over sleep deprivation, circadian rhythm, and caffeine with a minimal performance inhibitor model. Methods Enzymol. 2009;454:405-421.

85. Ramakrishnan S, Rajaraman S, Laxminarayan S, et al. A biomathematical model of the restoring effects of caffeine on cognitive performance during sleep deprivation. J Theor Biol. 2013;319:23-33.

86. Ramakrishnan S, Laxminarayan S, Wesensten NJ, Kamimori GH, Balkin TJ, Reifman J. Dose-dependent model of caffeine effects on human vigilance during total sleep deprivation. JTheor Biol. 2014;358: $11-24$

87. Puckeridge M, Fulcher BD, Phillips AJ, Robinson PA. Incorporation of caffeine into a quantitative model of fatigue and sleep. J Theor Biol. 2011;273(1):44-54.
Risk Management and Healthcare Policy

\section{Publish your work in this journal}

Risk Management and Healthcare Policy is an international, peer-reviewed open access journal focusing on all aspects of public health, policy, and preventative measures to promote good health and improve morbidity and mortality in the population. The journal welcomes submitted papers covering original research, basic science, clinical and epidemiological

\section{Dovepress}

studies, reviews and evaluations, guidelines, expert opinion and commentary, case reports and extended reports. The manuscript management system is completely online and includes a very quick and fair peerreview system, which is all easy to use. Visit http://www.dovepress.com/ testimonials.php to read real quotes from published authors. 